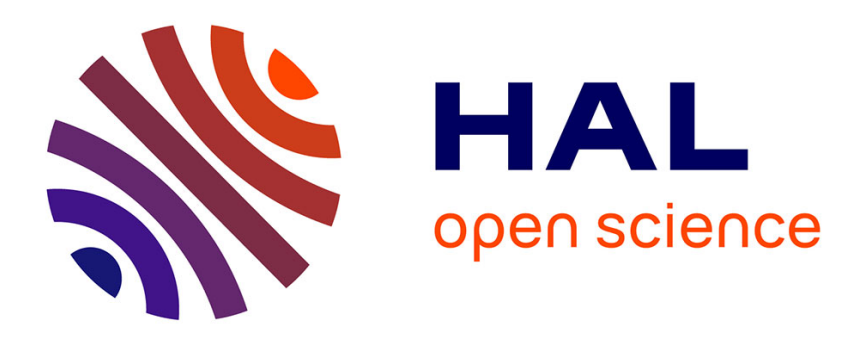

\title{
Sur la théorie thermodynamique de la capillarité et de l'électrocapillarité \\ M. Gouy
}

\section{To cite this version:}

M. Gouy. Sur la théorie thermodynamique de la capillarité et de l'électrocapillarité. J. Phys. Theor. Appl., 1901, 10 (1), pp.245-253. 10.1051/jphystap:0190100100024500 . jpa-00240501

\section{HAL Id: jpa-00240501 https://hal.science/jpa-00240501}

Submitted on 1 Jan 1901

HAL is a multi-disciplinary open access archive for the deposit and dissemination of scientific research documents, whether they are published or not. The documents may come from teaching and research institutions in France or abroad, or from public or private research centers.
L'archive ouverte pluridisciplinaire HAL, est destinée au dépôt et à la diffusion de documents scientifiques de niveau recherche, publiés ou non, émanant des établissements d'enseignement et de recherche français ou étrangers, des laboratoires publics ou privés. 


\section{SUR LA THÉORIE THERMODYNAMIQUE DE LA GAPLLARITÉ \\ ET DE L'ÉLECTROCAPILLARITÉ;}

Par M. GOUY.

1. Je me propose d'établir quelques propriétés des couches liquides superficielles, en vue des applications à la capillarité et à l'électrocapillarité. Je supposerai que les appareils ont de très petites dimensions dans le sens vertical, ou, si l'on veut, que l'expérience se fait dans un lieu où la pesanteur est nulle, afin de ne pas avoir à tenir compte de l'hétérogénéité des solutions due à la pesanteur (1). Le liquide est disposé de telle sorte que son volume et sa surface puissent varier indépendamment l'un de l'autre; il est, par exemple, maintenu par un anneau déformable qu'il mouille, et forme une lentille très aplatie $\left({ }^{2}\right)$. Les transformations considérées ont lieu à la température constante du milieu ambiant.

Pour fixer les idées, je considérerai d'abord le cas, du reste très important, du mercure immergé dans une solution. Le mercure est relié, avec interposition d'une force électromotrice, à une grande électrode (qui peut être aussi une électrode impolarisable). Cette électrode est telle que sa différence de potentiel avec la solution ne variera qu'avec la richesse de la solution et la pression qu'elle supporte.

J'admettrai que le faible courant qui existe à l'état de repos entre le mercure et la grande électrode, et qui est dû à la dépolarisation spontanée, constitue un phénomène accessoire qui peut être négligé ; cette hypothèse, nécessaire pour la réversibilité, est le point de départ de toute application de la thermodynamique aux questions de ce genre.

2. Au récipient de volume $u$, qui contient le mercure, la grande électrode et la solution sous la pression $P$, est adapté un corps de pompe fermé par une paroi perméable au dissolvant seul, qui contient le volume $v$ du dissolvant sous la pression $\mathrm{P}-p$, en désignant par $p$ la pression osmotique ( $f g .1$ ).

(1) Gocy et Chaperox, Annales de Chimie et de Physique. 6 série, t. XII.

(2) Assez épaisse cependant pour que la couche superficielle soit dans son état habituel. Il résulte aussi de cette disposition que la tension superficielle ne produit pas de pression hydrostatique, les surface étant sensiblement planes.

J. de Phys., $3^{\circ}$ série, t. X. (Avríl 1901.) 
Appelons S la surface du mercure; 0 , sa tension superficielle; V, la différence apparente de potentiel entre le mercure et la grande électrode; $q$, la quantité d'électricité donnée au mercure, à partir d'un état initial arbitraire. Prenons pour variables indépendantes $\mathrm{S}, \mathrm{V}, v, \mathrm{P}$.

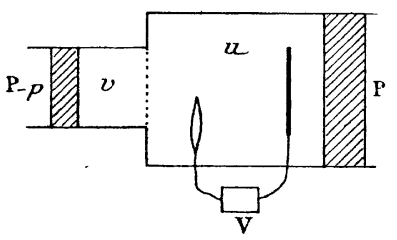

FIG. 1

L'opérateur, d'une manière réversible, fait varier $q, \mathrm{~S}, u$ et $v$ en fournissant les travaux $\mathrm{V} d q, \theta d \mathrm{~S},-\mathrm{P} d u$ et - $(\mathrm{P}-p) d v$. Les quantités $q$ et $u$ sont des fonctions des variables indépendantes $\left(^{1}\right)$. En développant $d q$ et $d u$, l'accroissement $d \varepsilon$ de l'énergie utilisable (travail de l'opérateur) (2) s'écrira

$$
\begin{aligned}
d \varepsilon=\left(\theta+\mathrm{V} \frac{\partial q}{\partial \mathrm{S}}-\right. & \left.\dot{\mathrm{P}} \frac{\partial u}{\partial \mathrm{S}}\right) d \mathrm{~S}+\left(\mathrm{V} \frac{\partial q}{\partial \mathrm{V}}-\mathrm{P} \frac{\partial u}{\partial \mathrm{V}}\right) d \mathrm{~V} \\
& +\left(p-\mathrm{P}+\mathrm{V} \frac{\partial q}{\partial v}-\mathrm{P} \frac{\partial u}{\partial v}\right) d v+\left(\mathrm{V} \frac{\partial q}{\partial \mathrm{P}}-\mathrm{P} \frac{\partial u}{\partial \mathrm{P}}\right) d \mathrm{P} .
\end{aligned}
$$

$d \mathrm{E}$ étant une différentielle exacte, il en résulte:

$$
\begin{aligned}
& \frac{\partial \theta}{\partial \mathrm{V}}=-\frac{\partial q}{\partial \mathrm{S}}, \\
& \frac{\partial 0}{\partial v}=\frac{\partial p}{\partial \mathrm{S}}, \\
& \frac{\partial p}{\partial \mathrm{V}}=-\frac{\partial q}{\partial v},
\end{aligned}
$$

$$
\begin{aligned}
& \frac{\partial \theta}{\partial \mathrm{P}}=\quad \frac{\partial u}{\partial \mathrm{S}}, \\
& \frac{\partial q}{\partial \mathrm{P}}=-\frac{\partial u}{\partial \mathrm{V}}, \\
& \frac{\partial p}{\partial \mathrm{P}}=1+\frac{\partial u}{\partial v} \text {. }
\end{aligned}
$$

3. En désignant par $\mathrm{C}_{s}$ la capacité du mercure, $\mathrm{S}$ variant seul, et par $\mathrm{C}_{\mathrm{V}}$ sa capacité, $\mathrm{V}$ variant seul, on a par définition :

$$
\mathrm{C}_{s}=\frac{\partial}{\partial \mathrm{S}} \quad \text { et } \quad \mathrm{C}_{\mathrm{V}}=\frac{\partial q}{\partial \mathrm{V}},
$$

(1) S'il n'en était pas ainsi pour $q$, on pourrait avoir, en revenant à l'état initial, $q \neq 0$, et, par suite, un résidu d'électrulyse qui ne pourrait manquer de modifier l'état du système.

(2) Je me sers ici de l'énergie utilisable qui abrège l'écriture (Journal de Phys., novembre 1889: mais on peut aisément établir les relations qui suivent par des cycles isothermes et réversibles. 
d'où résultent, d'après (1), les équations de M. Lippmann :

$$
\begin{aligned}
\mathrm{C}_{s} & =-\frac{\partial \theta}{\partial \mathrm{V}}, \\
\frac{\partial \mathrm{C}_{\mathrm{V}}}{\partial \mathrm{S}} & =\frac{\partial \mathrm{C}_{s}}{\partial \mathrm{V}}=-\frac{\partial^{2} \theta}{\partial \mathrm{V}^{2}} .
\end{aligned}
$$

4. Supposons maintenant que la solution ait été préparèe en mettant dans le récipient un poids $\pi$ d'un corps unique el un poids $\pi^{\prime} \mathrm{d} u$ dissolvant. Désignons par $r$ la richesse de la solution (') dans sun intérieur, c'est-à-dire abstraction faite des couches suparficielles. Si ces couches avaient la même constitution que l'intéricur. ot qu'aucune autre complication ne se produisît, on aurait $r=\frac{\pi}{\pi+\pi^{\prime}-\mathrm{D}\left(v-v_{0}\right)}$, en désignant par D le poids spécifique du dissolvant sous la pession $\mathrm{P}-p$, et par $v_{\mathrm{o}}$ la valeur initiale de $v$. En général, comme nous le verrons, la valeur exacte de $r$ sera un peu différente, et on peut toujours l'écrire ainsi :

$$
r=\frac{\pi+\alpha}{\pi+\alpha+\pi^{\prime}-\mathbf{D}\left(v-v_{0}\right)}
$$

$\alpha$ étant une quantité qui se présente sous la forme d'un accroissement positif ou négatif du poids du corps dissous. Cette quantité $\alpha$ est trop petite pour être mesurée directement, mais ses variations sont calculables et fournissent des données utiles au point de vue théorique.

Nous remarquons que l'état physique du système est déterminé quand $r, \mathrm{~S}, \mathrm{~V}, \mathrm{P}$ sont donnés; par suite, les fonctions de $r, \mathrm{~S}, \mathrm{~V}, \mathrm{P}$, que nous considérons ici, peuvent se mettre sous la forme $f(r, \mathrm{~S}, \mathrm{~V}, \mathrm{P})$; en particulier, $\theta$ est de la forme $f(r, \mathrm{~V}, \mathrm{P})$, et $p$ de la forme $f(r, \mathrm{P})$.

ว. Nous allons calculer les dérivées partielles $\frac{\partial x}{\partial S}$ et $\frac{\partial \alpha}{\partial V}$, en admettant que $r$ reste constant. Celte condition exige, quand $\mathrm{S}$ varie, que

(1) Poids dissous dans l'unité de poids de la solution. Le corps dissous peut être dissocié en jons ou de toute autre manière correspondant à un équilibre. J'admets, ici et par la suite, que le mercure n'entre nullement en solution. On peut concevoir, au contraire, qu'à l'état d'équilibre la solution contienne une très petite quantité d'ions ou de composés mercureux, fonction de $r$, $V$ et $P$; ce cas se ramène au précédent si l'on suppose que le mercure est enfermé aver une partie de la solution dans un vase dont la paroi est imperméable aux ions et composés mercureux et perméable à tout le reste. La richesse $r$ est alors relative à la partie de la solution qui est en dehors de cette cloison et qui est exempte de mercure. 
l'on ait:

$$
\frac{\partial r}{\partial \mathrm{S}} d \mathrm{~S}+\frac{\partial r}{\partial v} d v=0
$$

équation qui peut s’écrire :

$$
\frac{\partial p}{\partial \mathrm{S}} d \mathrm{~S}+\frac{\partial p}{\partial v} d v=0
$$

ou, d'après (2),

$$
\frac{\partial \theta}{\partial v} d \mathbf{S}+\frac{\partial p}{\partial v} d v=0
$$

et par suite:

$$
d v=-\frac{\frac{\partial \theta}{\partial v}}{\frac{\partial p}{\partial v}} d \mathbf{S}=-\frac{\frac{\partial \theta}{\partial r}}{\frac{\partial p}{\partial r}} d \mathbf{S}
$$

La variation correspondanle $d \alpha$ sera, d'après (10),

$$
d \alpha=-\frac{r}{1-r} \mathrm{D} d v=\frac{r}{1-r} \mathrm{D} \frac{\frac{\partial \theta}{\partial r}}{\frac{\partial p}{\partial r}} d \mathrm{~S} .
$$

Il vient donc:

$$
\frac{\partial \alpha}{\partial \mathrm{S}}=\frac{r}{1-r} \mathrm{D} \frac{\frac{\partial \theta}{\partial r}}{\frac{\partial p}{\partial r}}
$$

6. Comme $\alpha$ est fonction de $r, \mathrm{~S}, \mathrm{~V}, \mathrm{P}$, on peut écrire, d'après (11)

$$
\frac{\partial}{\partial \mathrm{S}}\left(\frac{\partial \alpha}{\partial \mathrm{V}}\right)=\frac{\partial}{\partial \mathrm{V}}\left(\frac{\partial \alpha}{\partial \mathrm{S}}\right)=\frac{r}{1-r} \mathrm{D} \frac{\frac{\partial}{\partial \mathrm{V}}\left(\frac{\partial \theta}{\partial r}\right)}{\frac{\partial p}{\partial r}}=\frac{r}{1-r} \mathrm{D} \frac{\frac{\partial}{\partial r}\left(\frac{\partial \theta}{\partial \mathrm{V}}\right)}{\frac{\partial p}{\partial r}} .
$$

Mais le second membre est indépendant de $\mathrm{S}$, et il vient, d'après (8),

$$
\frac{\partial \alpha}{\partial \mathbf{V}}=-\mathrm{SD} \frac{r}{1-r} \frac{\frac{\partial \mathrm{C}_{s}}{\partial p}}{\frac{\partial p}{\partial p}}+\mathbf{B},
$$

B désignant une quantité indépendante de $\mathrm{S}$. 
L'équation (3) nous donne, par un calcul analogue à celui du $n^{\circ} 3$,

$$
\frac{\partial \mathrm{x}}{\partial \mathrm{V}}=-\frac{r}{1-r} \mathrm{D} \frac{\frac{\partial q}{\partial r}}{\frac{\partial p}{\partial r}} .
$$

En comparant (12) et (13), il vient :

$$
\mathrm{B}=\frac{r}{1-r} \frac{\mathrm{D}}{\frac{\partial p}{\partial r}}\left(\mathrm{~S} \frac{\partial \mathrm{C}_{s}}{\partial r}-\frac{\partial q}{\partial r}\right) .
$$

Ces formules seront encore applicables, même si $v$ demeure invariable, lorsque la solution sera assez abondante pour que les variations de $r$ soient négligeables, ce qui aura toujours lieu dans les conditions ordinaires.

7. Les formules précédentes ne supposent pas nécessairement que le mercure reste pur. Si la solution contient un composé d'un métal M, le mercure, à l'état d'équilibre, que nous considérons ici, deviendra, en général, un amalgame liquide du métal $M$, d'autant plus riche qu'on approchera davantage de la valeur de $\mathrm{V}$ qui donne une électrolyse continue. Cette limite correspond, comme on sait, au point où, le mercure étant saturé du métal $\mathrm{M}$, il commence à se former un amalgame solide. En deçà de cette limite, l'équilibre correspond, pour chaque valeur de $\mathrm{V}$, à une richesse déterminée $r_{a}$ de l'amalgame liquide, qui devient très pelite et insensible à l'analyse, quand on est quelque peu éloigné de la limite, mais dont on ne peut faire abstraction au point de vue théorique.

D'après (12), dansl'expression de $\frac{\partial \alpha}{\partial V} d V$, le terme $\mathrm{B} d \mathrm{~V}$, indépendant de S, exprime l'effet produit sur la solution par la fraction du courant qui est employée à nodifier la richesse de l'amalgame. Ce terme $\mathrm{B} d V$ sera nul si le mercure reste pur, ou bien si la grande électrode est formée du métal M ; dans ce dernier cas, en effet, cette fraction du courant effectue un transport du métal $M$ entre la grande électrode et le mercure, sans gain ni perte pour la solution. On a alors, d'après (14),

$$
\frac{\partial q}{\partial r}=\mathrm{S} \frac{\partial \mathrm{C}_{s}}{\partial r}
$$


relation qui nous montre que, $r$ variant seul, le courant qui en résulte produit seulement un effet superficiel, indépendant du volume du mercure. Ainsi, dans ces conditions, $r_{a}$ ne dépend pas de $r$, mais seulement de $\mathrm{V}$ et de $\mathrm{P}$, résultat déjà connu $\left(^{1}\right)$.

8. D'après les équations (11) et (12), l'accroissement de $\mathrm{S}$ ou de V agit sur la richesse $r$, comme le ferait l'addition, à la solution supposée seule, d'un certain poids du corps dissous, positif ou négatif. Comme $\frac{\partial p}{\partial r}$ est toujours positif, on voit qu'un accroissement positif $d \mathrm{~S}$ enrichit ou appauvrit la solution, suivant que $\frac{\partial \theta}{\partial r}$ est positif ou négatif; cet effet ne peut résulter que d'actions produites aux surfaces du mercure et de la grande électrode.

Considérons d'abord le cas simple où $\mathrm{V}$ est tel que le mercure possède son maximum de tension superficielle. Alors, d'après l'équation (8), la variation de $\mathrm{S}$ ne produit pas de courant, et la grande électrode est hors de cause. D'autre part, la surface mercurielle et la couche voisine restent dans le même état physique, et l'éte due de la surface varie seule. Il faut donc qu'il existe, dans la couche voisine de la surface mercurielle, ou un appauvrissement de la solution $\left(\frac{\partial \theta}{\partial r}>0\right)$, ou une accumulation du corps dissous $\left(\frac{\partial \theta}{\partial r}<0\right)\left({ }^{2}\right)$. La quantité du corps dissous qui se trouve ainsi ajoutée ou sous-

traite au reste de la solution est, en valeur absolue, $\mathrm{D} \frac{r}{1-r} \frac{\frac{\partial \theta}{\partial r}}{\frac{\partial p}{\partial r}}$ par unité de surfaçe. Cette quantité, toujours fort petite, peut atteindre, d'après mes mesures de $\frac{\partial \theta}{\partial r}$, quelques millionièmes de milligramme par centimètre carré, c'est-à-dire l'ordre de grandeur des couches ioniques produites par la polarisation électrolytique.

9. Si $\theta$ n'est pas à son maximum, la variation de $\mathrm{S}$ fait varier $q$,

(1) D’après un théorème connu, la force électromotrice d'une pile dont les deux pôles sont un amalgame de $\mathbf{M}$ et ce métal lui-même est indépendante de la solution employée, pourvu qu'elle contienne un composé de $\mathbf{M}$.

$(\because)$ On remarquera que l'effet est d'un sens tel qu'il diminue la tension superficielle. Le résultat ci-dessus a été indiqué aux Comples Rendus du 3 décembre 1900. Pour les faits dexpérience, je renverrai le lecteur aux notes publiées dans les Comptes Rendus des 30 juillet et 19 novembre 1900. 
et une électrolyse a lieu, dont il faut tenir compte pour interpréter les variations de $\alpha$. Je me bornerai ici à éclaircir ce point par un exemple.

Admettons la théorie de l'électrocapillarité d'Helmholtz, où l'électrolyse seule peut faire varier $r$. La grande électrode est supposée polarisable (1) et positive par rapport à l'électrolyte, et il existe à sa surface, dans l'électrolyte, une couche d'anions. Si le mercure est aussi positif, la couche électrique de l'électrolyte à son contact est aussi formée d'anions. Le courant produit par la variation de S a pour effet final de transporter des anions d'une surface à l'autre, sans autre changement, et, par suite, la richesse $r$ ne variera pas. Ainsi, dans ce cas, $\frac{\partial \alpha}{\partial S}$ est nul, et de même $\frac{\partial \theta}{\partial r}$, d'après $(\mathbf{1 1})$.

$\mathrm{Si}$, au contraire, le mercure est négatif par rapport à l'électrolyte, la couche superficielle est formée de cathions. Le courant produit par un accroissement positif $d \mathrm{~S}$ augmente le nombre de ces cathions, et aussi le nombre des anions de la grande électrode ; la solution est ainsi appauvrie, $\frac{\partial \alpha}{\partial \mathrm{S}}$ est négatif et de même $\frac{\partial \theta}{\partial r}$.

Si donc on trace les courbes électrocapillaires (V abscisse, $\theta$ ordonnée), pour $r$ et $r+d r$, la seconde coüncide avec la première par sa branche positive, et si $d r>0$, elle est au-dessous de la première par sa branche négative. Sur les deux branches négatives prenons deux points où $\theta$ a la même valeur; soit $d V$ la différence de leurs abscisses. Nous aurons :

$$
\frac{\partial \theta}{\partial r} d r+\frac{\partial \theta}{\partial V} d \mathbf{V}=0
$$

ou bien, d'après (11),

$$
\frac{\partial \alpha}{\partial \mathrm{S}} \frac{\partial p}{\partial r} \frac{1-r}{\mathrm{D} r} d r+\frac{\partial \theta}{\partial \mathrm{V}} d \mathrm{~V}=0 .
$$

Mais, dans l'hypothèse que nous examinons, $\frac{\partial \alpha}{\partial S}$ doit être proportionnel à $\frac{\partial q}{\partial S}$, du moins si l'on admet que les ions s'accumulent aux

(1) J'entends par là que ce n'est pas une électrode impolarisable, mais bien une lame de platine, par exemple. 
surfaces et sont ainsi soustraits au reste de la solution; il en résulte donc, d'après $(\mathbf{1}), d \mathrm{~V}=\mathrm{C}^{\mathrm{te}}$.

La considération de $\frac{\partial \alpha}{\partial V}$ donne le même résultat. Ainsi les branches négatives des deux courbes seront superposables par une translation parallèle à l'axe des $\mathrm{V}$.

Il résulterait donc de cette hypothèse que la largeur de la courbe à toute hauteur varierait en sens inverse de la richesse de la solution, d'une quantité indépendante de la hauteur considérée $\left(^{1}\right)$. J'ai constaté en effet que la première partie de cet énoncé est toujours vérifiée par l'expérience, sauf au voisinage du maximum; mais il n'en est pas de même de la seconde partie, qui, du reste, n'est pas admissible en toute rigueur.

10. L'équation (4) nous montre que, si $\theta$ varie avec $P$, le volume $u$, qui comprend la solution, le mercure et la grande électrode, varie avec S. Cette variation de volume peut résulter, en général, des actions envisagées plus haut; mais elle prend une signification plus nette dans le cas où la solution n'est autre que le dissolvant pur. En faisant l'expérience au maximum de $\theta$, pour éviter les difficultés d'interprétation dues à l'électrolyse, on voit que, si $\frac{\partial \theta}{\partial \mathrm{P}} \neq 0$, il en résultera que soit pour le mercure, soit pour le liquide ambiant, la couche superficielle a une densité différente de sa valeur normale, comme on l'a quelquefois supposé sans vérification expérimentale.

L'équation (5) nous indique que, si l'on fait varier seulement $\mathrm{P}$, il peut se produire un courant de courte durée entre le mercure et la grande électrode $\left({ }^{2}\right)$.

L'équation (6) est étrangère à notre sujet $\left({ }^{3}\right)$.

11. Reprenons maintenant l'appareil figuré plus haut, et supposons que, la grande électrode étant supprimée, le mercure soit isolé. Les équations (2) et (4) sont encore exactes, ainsi que les formules qui s'en déduisent; mais la valeur des coefficients différentiels n'est plus la même. Toutefois les deux problèmes sont identiques, si l'on est

(1) Si l'on suppose que la grande électrode est négative par rapport à la solution, ou bien impolarisable, on retrouve naturellement les mêmes relations de forme entre les deux courbes.

${ }^{(2)}$ Ces deux expériences sont en préparation.

(3) Cette équation montre que la pression osmotique dépend, en général, de la pression ambiante, relation déjà établie ailleurs (Govy et Chaperon, Sur l'équilibre osmotique, Annales de Chimie et de Physique, janvier 1888). 
au maximum de \%, car la grande électrode ne joue alors aucun rôle. Enfin on peut supposer que le mercure est remplacé par un autre liquide non miscible avec celui du récipient.

12. Un cas assez important est celui où le mercure est remplacé par un gaz ou par la vapeur seule de la solution; mais il convient de le traiter un peu autrement. Prenons, pour variables indépendantes, S, $u$ et $v$; la variation de l'énergie utilisable sera:

$$
d \varepsilon=\theta d \mathrm{~S}-\mathrm{P} d u-(\mathrm{P}-p) d v\left({ }^{1}\right),
$$

d'où l'équation:

$$
\frac{\partial \theta}{\partial v}=\frac{\partial(p-\mathrm{P})}{\partial \mathrm{S}}
$$

qui donne, par un calcul analogue à celui du $n^{\circ} 5$,

$$
\frac{\partial \alpha}{\partial \mathrm{S}}=\mathrm{D} \frac{r}{1-r} \frac{\frac{\partial \theta}{\partial r}}{\frac{\partial(p-\mathrm{P})}{\partial r}} .
$$

Ici $\partial \frac{(p-\mathrm{P})}{\partial r}$ est positif comme $\frac{\partial p}{\partial r}$, dont il diffère peu. La variation de $\alpha$, quand $\mathrm{S}$ varie, peut provenir de deux causes $: 1^{\circ}$ la couche superficielle de la solution n'a pas la même richesse que l'intérieur, comme précédemment;

$2^{\circ}$ La couche superficielle de gaz ou de vapeur est différente du gaz ou de la vapeur. Cette seconde cause n'existe pas, quand le dissolvant et le corps dissous ne sont volatils nil'un ni l'autre, et on peut regarder comme probable qu'elle est négligeable, en général. D’après cela, les solutions qui ont une tension superficielle variable avec leur richesse (eau et alcool, par exemple) doivent avoir leur couche superficielle plus ou moins riche que l'intérieur, le sens de cette différence étant toujours tel que la tension superficielle en soit diminuée.

(1) Dans les cas que nous examinons, il arrivera souvent que $\mathbf{P}-p$ sera négatif; mais il n'y a pas là de difficulté, les liquides pouvant supporter des pressions négatives considérables. 